TITLE:

\title{
Information-Theoretic Characterization of Brain Registration and Structure- Function Relationships
}

\author{
AUTHOR(S): \\ Yamamoto, Hiroki
}

\section{CITATION:}

Yamamoto, Hiroki. Information-Theoretic Characterization of Brain Registration and Structure-Function Relationships. Neuroscience and Biomedical Engineering 2016, 4(2): $140-147$

\section{ISSUE DATE:}

2016-09

URL:

http://hdl.handle.net/2433/216513

\section{RIGHT:}

This is an Open Access Article. 


\title{
Information-Theoretic Characterization of Brain Registration and Struc-
} ture-Function Relationships

\author{
Hiroki Yamamoto ${ }^{\mathrm{a}_{*}}$
}

\author{
${ }^{a}$ Human and Environmental Studies, Kyoto University, Kyoto, Japan
}

\section{A R T I C L E H I S T O R Y \\ Received: April 19, 2016 \\ Revised: June 21, 2016 \\ DOI: \\ $10.2174 / 221338520466616062808$ \\ 3230}

\begin{abstract}
Background: In functional brain imaging, intersubject brain registration is widely used to describe the loci of brain activation or lesions and to normalize functional data between individual brains based on anatomical similarities. However, such registration necessarily has limits because brain structure varies among individuals and is not always closely correlated with brain function.

Objective: This study quantitatively compared three registration algorithms - linear volume-based, nonlinear volume-based, and surface-based methods-using probability and entropy maps of human visual areas.

Methods: fMRI retinotopic mapping was performed in 16 subjects to construct a model for 12 visual areas. The surface and volumetric models of each visual area were registered to the standard brain template using the three registration methods.

Results: After surface-based registration, the probability of visual areas being present in the common space was increased approximately 3 -fold compared with the volume-based method, but the average probability was relatively small at approximately 0.3 . On the other hand, average entropy was around 1 bit, revealing no significant difference between the two methods.

Conclusion: Our results indicate that the current technology has room for improvement and thus should be used carefully with consideration of its limits. We suggest that the information-theoretic approach can be naturally extended to the analysis of brain structure-function relationships by taking advantage of mutual information.
\end{abstract}

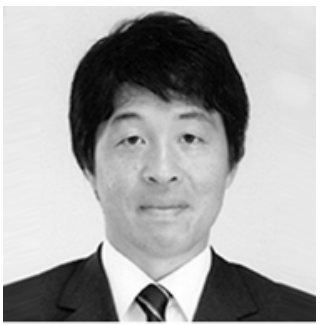

Hiroki Yamamoto

Keywords: Visual cortex, fMRI, probabilistic atlas, brain normalization, entropy.

\section{INTRODUCTION}

The human cerebral cortex consists of multiple functionally distinct areas, many of which have recently been identified by structural and functional imaging. The locations of different functional areas can be quantitatively compared between individuals once the areas are expressed in a common coordinate space using the same brain template. Many of the various techniques proposed for intersubject brain registration are currently being used to locate the loci of brain activation or lesions as well as to reveal intersubject differences in functional brain data [1]. Therefore, it is important for reliable interpretation of functional brain imaging data that the efficiencies of the different registration methods should be comparatively evaluated.

We have previously proposed an information-theoretic analysis method for evaluating brain registration techniques which is based on the concepts of "inconsistency" and "uncertainty" $[2,3]$. Inconsistency is defined as a difference in the location of a particular visual area between individuals

*Address correspondence to this author at the Department of Human \& Environmental Studies, Kyoto University, Kyoto, Japan; Tel/Fax: +81-75753-2978;+81-75-753-6574; E-mail: yamamoto@cv.jinkan.kyoto-u.ac.jp after registration. This concept has been widely applied to the investigation of interindividual variability in functional areas and the efficacy of intersubject registration methods. Inconsistency can be quantitatively analyzed by constructing a probability map [4-6], a map that expresses the probability of a particular visual area being present at a certain point in the common space (how many people among a given population have that particular functional area in the same location).

Although inconsistency is a useful index for analyzing a single isolated area, it is not sufficient for studying multiple areas. When multiple areas immediately adjacent to each other are simultaneously expressed in a common space, functionally distinct areas may overlap between individuals. If this happens, it is difficult to predict which point in the space corresponds to which visual area, thus generating uncertainty about the identity of visual areas. Uncertainty increases as the number of overlaps increases. If activation or a lesion occurs in an area of high uncertainty, accurate identification of functional areas will be difficult even when the inconsistency is small. In a multi-subject group analysis of functional data, attention must be paid to the contamination of responses from multiple functional areas located in the area of high uncertainty. We previously incorporated entropy 
[7], a concept in information theory, as a measure of uncertainty to construct an entropy map, a map constructed using the probability of multiple functional areas being present at a certain point in the common space.

The present study aimed to evaluate the efficiency of registration algorithms using probability and entropy maps. The registration algorithms currently available are roughly divided into those that are volume-based and those that are surface-based [8]. The volume-based algorithms use the distribution of voxel intensities in the three-dimensional structure of the cerebrum to register individual brains, via either a linear or nonlinear transformation, into the standard brain template. By contrast, the surface-based algorithms use structures on the cortical surface (sulci and gyri) of an individual brain to match them to the structures on the standard template. To match the cortical structures, specific landmarks or measurement values such as mean curvature are used. In this study, we used the human visual cortical areas determined by functional magnetic resonance imaging (fMRI) to compare the surface-based method with the linear and nonlinear volume-based methods. We also discussed the possibility that our information-theoretic approach could be naturally extended for the analysis of brain structurefunction relationships by taking advantage of mutual information.

\section{MATERIALS AND METHODS}

\subsection{Surface and Volumetric Models of the Visual Cortex}

fMRI retinotopic mapping was performed in 16 subjects ( 2 women, $14 \mathrm{men}$; 32 hemispheres) to construct a model for 12 visual areas (V1, V2d, V2v, V3d, V3v, V3A, V3B, V7, LOc, MT+, V4v, and V8) in each hemisphere (Fig. 1). First, a model of the cortical surface of individual brains was reconstructed from structural MR images. Phase-encoded retinotopy measurements [9-13] were then performed to identify the boundaries of the visual areas on the cortical surface, which were then used to excise the surface models of the visual areas from the cortical surface. Lastly, the corresponding volumetric model was constructed by adding $3-\mathrm{mm}$ thickness over the surface model, as described in detail in our previous study [14].

\subsection{Intersubject Brain Registration}

The surface and volumetric models of each visual area were registered to the standard brain template using the following three registration methods.

Linear volume-based method. The volumetric model of the visual areas was registered to Talairach space [15] using the linear volume-based method with nine parameters consisting of translation, rotation, and orthogonal scaling each around the $\mathrm{X}-, \mathrm{Y}-$, and $\mathrm{Z}$-axes. The origin of the space was the upper end of the anterior commissure. The $\mathrm{X}$-axis represents the sagittal plane, the Y-axis the coronal plane, and the $\mathrm{Z}$-axis the horizontal plane. To determine the transformation matrix, we identified the locations of the anterior commissure, posterior commissure, and inner wall according to the conventional method [16], as described in our previous report [14].

Nonlinear volume-based method. The volumetric model of the visual areas was registered to the common space using a nonlinear transformation. First, the skull was eliminated from the anatomical images [17]. Then, the brain images were registered to the Colin27 brain template [18] using the automated nonlinear transformation function of AIR software [19]. This method involves an affine transformation (12 parameters), followed by a second-order nonlinear polynomial transformation (30 parameters) to match an individual brain to the standard template. Lastly, using best-fit parameters, the volumetric model of the visual areas was registered to the common space.

Surface-based method. Automated spherical warping [20] was used to register the surface model of the visual areas. In this method, the cortical sulci and gyri in an individual brain were automatically registered to the FreeSurfer fsaverage brain [21] using the information on curvature.

\subsection{Construction of the Probability Map}

We created the probability map by calculating the probability of each visual area being present at a certain space or point on the standard brain template (Fig. 2). The number of subjects with a particular visual area at a certain anatomical space or point was divided by the total number of subjects (16) to obtain the probability. In addition, the maximum probability map was obtained after identification of the area with the greatest probability of being at a particular anatomical point.

\subsection{Construction of the Entropy Map}

As shown in (Fig. 2), for every point in the space or on the surface of the standard template, the Shannon entropy $H$ was calculated from the probability of each visual area $p_{i}$ using the formula

$$
H=-\sum_{i=1}^{n} p_{i} \cdot \log _{2} p_{i} \text { [bits]. }
$$

Here, $n$ was 13 as the non-visual area $p_{13}$ was added to the visual areas $p_{1} \sim p_{12}$. The upper limit of the entropy is $\sim 3.7$ bits when the probabilities of all of the visual areas become similar; in other words, when no prediction can be made as to which area is present.

\subsection{Statistical Analysis}

The R package (R Development Core Team 2016) was used for all analyses. The probability and entropy maps were statistically analyzed at the level of visual areas by averaging all of the probability or entropy values within each area $\left(p_{i}>\right.$ 0 ). The effects of the three registration methods on the maps were compared by one-way repeated measures analyses of variance (ANOVAs) across the areas, followed by t-tests with multiple comparison using Shaffer's modified Bonferroni correction. For each ANOVA, the Greenhouse-Geisser epsilon was used to correct for violations of sphericity. To assess the reproducibility of the average probability and entropy values, we divided subjects randomly into two groups and constructed probability and entropy maps for each group. Next, the areal average probability and entropy values were obtained for the map of each group. Finally, correlations between the split-samples of the areal average values were assessed by Pearson's correlation analysis. 
S1 Left

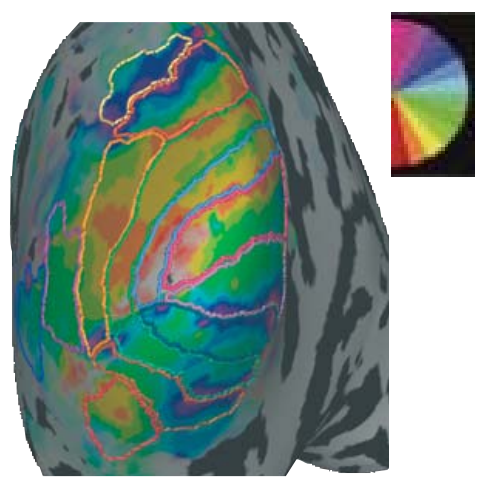

S1 Right

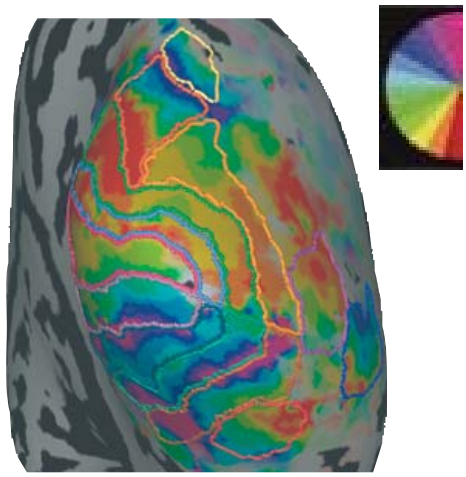

V1d V2d V3dV3A V7 V3B LOc MT+V1v V2v V3v V4v V8

Fig. (1). Locations of retinotopic areas, V1d/v, V2d/v, V3d/v, V3A, V3B, V7, V4v, V8, LOc, and MT+, in one subject's (S1) hemispheres and their relation to visual field representation (up to $16^{\circ}$ eccentricities). The colored lines on the inflated cortices indicate the border of each area by the color code below the hemispheres. The color overlay on the cortex indicates the preferred stimulus angle at each cortical point by the color code to the right of the hemispheres. The more saturated the color, the higher the statistical significance of retinotopic activity. This retinotopy was measured by carrying out phase-encoding fMRI while the subject viewed a wedge-shaped colored checkered pattern rotated around the fixation point.

\section{A}

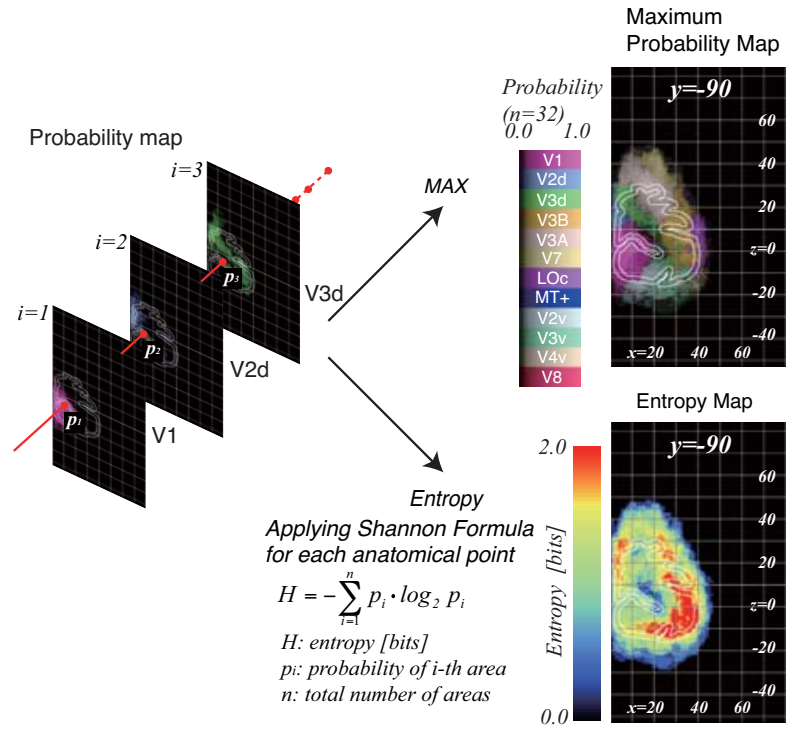

$\mathrm{B}$

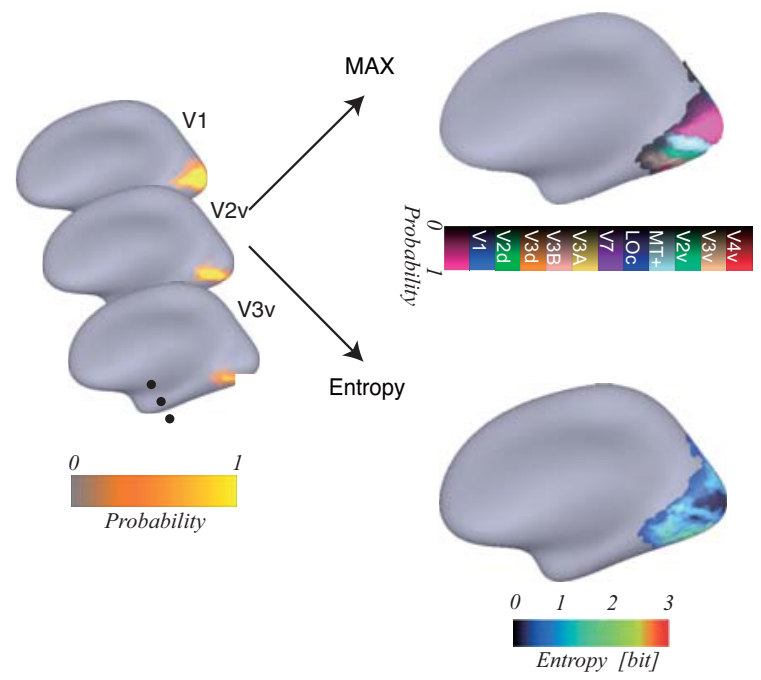

Fig. (2). (a) Volume-based method. Left: Probabilistic maps of V1, V2v, and V3d, etc. from 16 subjects in Talairach space on a coronal section $(y=-90)$. The probability that each area is located at each voxel is color coded and superimposed on the outline drawing of the Talairach brain showing the cortical gray matter. Upper right: Maximum probability map of V1, V2v, and V3d, etc. on the section. The visual area with the most occurrences and its occurrence probability (i.e., maximum probability) for each voxel are color-coded, as shown by the color bar. The occurrence probability of a given area was calculated by dividing the number of overlapping hemispheres by the total number of samples, all of which were aligned into the space using global linear or nonlinear transformations. Lower right: Entropy map of V1, V2v, and V3d, etc. on the section. The entropy at each voxel is color-coded, as shown by the color bar. The entropy was computed from the probability maps of visual areas using the Shannon formula shown in the lower middle. (b) Surface-based method. Left: Probabilistic maps of V1, V2v, and V3v, etc. from 16 subjects in medial views of the inflated FreeSurfer average right hemisphere. The probability that each area is located at each surface vertex is represented by a color code, as shown by the color bar. The probability of a given area was calculated by dividing the number of overlapping hemispheres by the total number of samples, all of which were aligned on the average surface using spherical morphing. Upper right: The same view of the maximum probability map. The most probable visual area and its occurrence probability $(\mathrm{n}=16)$ for each surface vertex are color-coded using different colors and brightness levels, respectively, as indicated by the color bar. Lower right: the same view of the entropy map. The entropy for each vertex is color-coded, as shown by the color bar. 


\section{RESULTS}

The 12 visual areas were successfully identified for all 32 hemispheres. We then constructed the probability and entropy maps of the visual areas using the three registration methods mentioned above. Maps were three-dimensional volume or surface data in the FreeSurfer or Visualization Toolkit file format and could be viewed using in-house software called BAIVEW ([22]; http://www.cv.jinkan.kyotou.ac.jp/site/resources/) or an open source visualization application, such as ParaView (http://www.paraview.org/).

(Fig. 3) shows the slice representation of a part of the probability and entropy maps constructed using the nonlinear volume-based method. The maximum probability and entropy maps were overlaid onto the brain in the Talairach atlas (white outline). (Fig. 4) shows the results of the surfacebased registration, where both maps were overlaid on the inflated version of the FreeSurfer brain template.

\subsection{Inconsistency in the Location of Human Visual Ar- eas: Probability Map}

Similar to those observed in individual brains, visual areas with the maximum probability value were arranged in the order of lower to higher. However, in each visual area, the probability map was not confined to a small area, but rather spread far beyond the projected range. This suggests the presence of large intersubject variability in the locations of the visual areas. To quantitatively analyze the degree of inconsistency, we calculated the mean probability for each visual area to compare the values between the registration methods and between the visual areas (Fig. 5A).

The mean probability value was much higher with the surface-based method than with the volume-based method, suggesting that the visual areas in individual brains fit well in the common space when the surface-based method is used, reducing inconsistency. The comparison of the two volumebased registration methods revealed that the nonlinear method was slightly superior to the linear method. These results were statistically confirmed by a one-way repeated measures ANOVA, which showed a main effect of the registration method $\left(F_{(2,22)}=118.05, p<0.001\right)$. Post hoc t-tests showed significant differences between the surface- and nonlinear volume-based methods $(t=10.11, p<0.001)$, the surfaceand linear volume-based methods $(t=11.71, p<0.001)$, and the nonlinear and linear volume-based methods $(t=7.57, p<$ $0.001)$. For all visual areas, the means and standard deviations (SDs) were 0.27 (0.074) for the surface-based method, $0.10(0.013)$ for the nonlinear volume-based method, and $0.089(0.016)$ for the linear volume-based method.

Mean probability values varied slightly among the different visual areas, with V1 having the maximum value regardless of the method used. To investigate if such dependency on the visual areas was reproducible, we calculated the mean probability by randomly dividing subjects into two groups or comparing the left and right hemispheres. (Fig. 5B) shows a parallel coordinate plot obtained using the nonlinear volumebased method. The dependence on the visual area was hardly changed even after dividing the subjects into two groups (correlation coefficient between the groups: 0.826, $p=9.4 \times 10^{-4}$; correlation coefficient between the hemispheres:
0.787, $\left.p=2.4 \times 10^{-3}\right)$. High reproducibility was also observed with the other methods.

\subsection{Uncertainty in the Location of Human Visual Areas: Entropy Map}

We analyzed uncertainty among individual visual areas in the same manner as for inconsistency, by comparing the mean entropy values between the three registration methods and between the visual areas (Fig. 5C).

Mean entropy values obtained using the surface-based method showed that V1 was smaller and other areas were larger than those obtained using the volume-based methods. This suggests that uncertainty in the visual areas, except for $\mathrm{V} 1$, in the common space increases when the surface-based method is used. The comparison of the two volume-based methods revealed that all visual areas except for V1 and V8 were larger in the nonlinear method than in the linear method. A one-way repeated measures ANOVA confirmed the difference in entropy values across the registration methods, indicating a significant main effect of the method $\left(F_{(2,22)}\right.$ $=9.55, p=0.0062$ ). Post hoc t-tests showed significant differences between the surface- and nonlinear volume-based methods $(t=2.36, p=0.038)$, the surface- and linear volume-based methods $(t=3.40, p=0.0059)$, and the nonlinear and linear volume-based methods $(t=3.67, p=0.0037)$. For all visual areas, the means and SDs were as high as 1.15 bits $(0.25)$ for the surface-based method, followed by $1.07(0.20)$ for the nonlinear method and $1.01(0.18)$ for the linear volume-based method.

Mean entropy values were also dependent on the visual areas. The parallel coordinate plot shown in (Fig. 5D) reveals that the visual areas can be divided into three major classes based on the magnitude of entropy, and this trend was reproduced even after dividing subjects into two groups (correlation coefficient between the groups: $r=0.877$, $p=2.1 \times 10^{-9}$; correlation coefficient between the hemispheres: $\left.r=0.955, p=9.6 \times 10^{-9}\right)$. High reproducibility was also observed with the other methods.

\section{DISCUSSION}

\subsection{Inconsistency in the Location of Human Visual Ar- eas: Probability Map}

In the common space, both linear and nonlinear volumebased methods gave a probability of approximately 0.1 , with no significant difference between the methods. By contrast, the mean value for the surface-based method was almost 0.3 and thus approximately 3 -fold that of the volume-based methods. These results are consistent with the reports of previous studies $[6,20,23]$ and thus indicate the superiority of the surface-based method over the volume-based methods. However, given the relatively small sample size of the present study, definite conclusions should not be drawn. Comparisons of the visual areas revealed that V1, which is located over the calcarine sulcus, had the smallest inconsistency and a mean probability of approximately 0.5 with the surface-based method, a value similar to that obtained by the same method in a study using V1 identified based on the cellular architecture [24]. This suggests that fMRI-based identification of the visual areas is extremely accurate. 
A
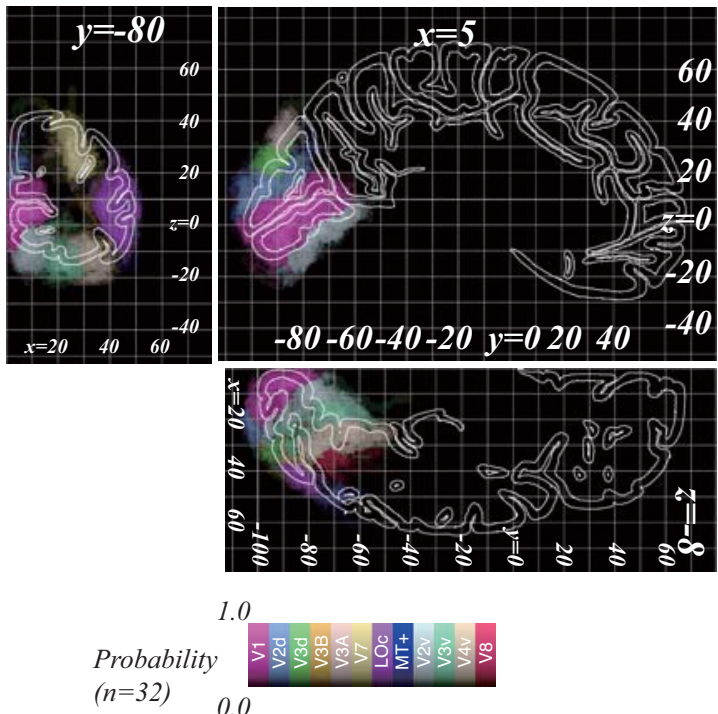

$\mathrm{B}$
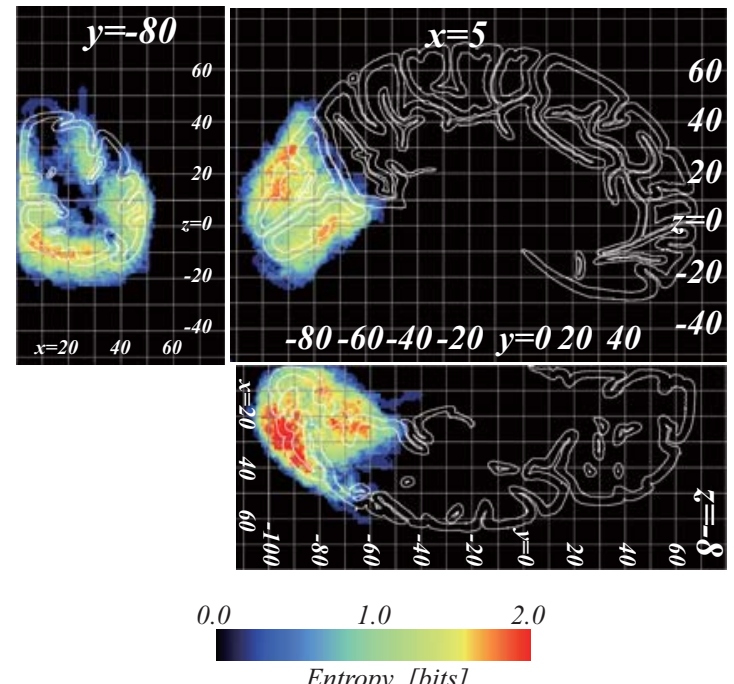

Entropy [bits]

Fig. (3). Probability and entropy maps of human visual areas constructed using nonlinear volume-based registration. (a) 3D maximum probability maps of visual areas, V1d/v, V2d/v, V3d/v, V3A, V3B, V7, V4v, V8, LOc, and MT+, in Talairach space on coronal (upper left), sagittal (upper right), and axial sections (lower right). The visual area with the most occurrences and its probability (i.e., maximum probability) for each voxel are color-coded using 13 different colors and 10 brightness levels, respectively (see the color bar in the bottom), and are superimposed on the outline drawing of the Talairach brain. (b) 3D entropy map of the visual areas on the sections. The entropy for each voxel is color-coded, as shown by the color bar.

A

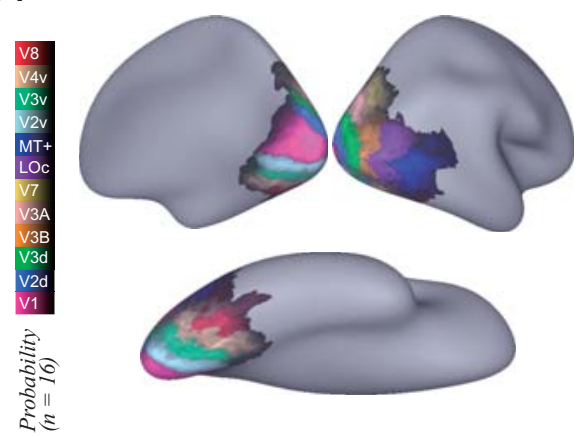

B

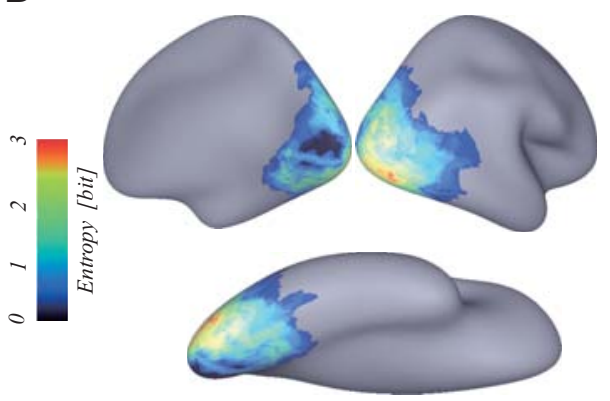

Fig. (4). Probability and entropy maps of human visual areas constructed using surface-based registration. (a) Maximum probability surface maps of visual areas, V1d/v, V2d/v, V3d/v, V3A, V3B, V7, V4v, V8, LOc, and MT+, on the inflated FreeSurfer average right hemisphere. The probability that each area is located at each surface vertex is represented by a color code, as shown by the color bar. Upper left: medialposterior view. Upper right: lateral-posterior view. Bottom: ventral view. (b) Entropy surface map of the visual areas. The entropy for each vertex is color-coded, as shown by the color bar. Upper right: lateral-posterior view. Bottom: ventral view.

\subsection{Uncertainty in the Location of Human Visual Areas: Entropy Map}

In the comparisons of mean entropy values, the superiority of the surface-based method was observed for V1, but entropy tended to increase for other visual areas. While somewhat surprising, this finding can be explained by two factors. The first is the discrepancy between anatomical points used for the mean calculation. While points on the cortical surface were used with the surface-based method, points on the cortical surface as well as in nearby regions were used with the volume-based methods. Entropy values may be increased if the calculation is performed using only the points on the cortical surface in all subjects. The second factor is the discrepancy in dimension. The surface-based method investigated the degree of overlap between different visual areas within the two-dimensional representation of the cortical surface, whereas the volume-based methods assessed overlaps within the three-dimensional representation of the visual cortex (3-mm thickness). This means that in the surface-based method the common space is densely crowded with visual areas, readily creating overlaps. If this is taken into consideration, given that the surface-based method showed entropy values increased by approximately 30\% while that of V1 was reduced, the surface-based method appears to be superior to the volume-based methods. It is necessary therefore to keep the discrepancy in dimension in mind when comparing the efficiency of volume-based and surface-based registration methods. 

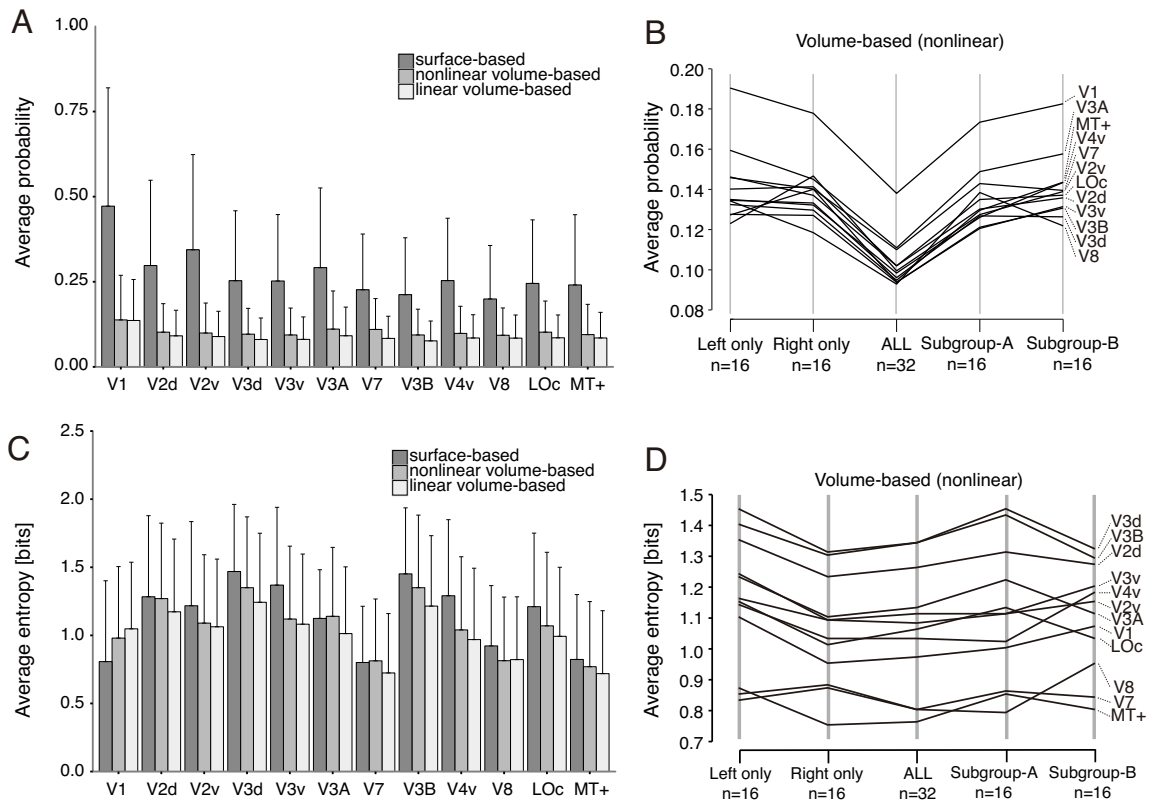

Fig. (5). Post-registration mean probability and entropy values. (a) Mean probability values for the different registration methods and the dependency on visual areas. Error bars denote standard deviation. (b) Reproducibility of the visual area dependency of mean probability. (c) Mean entropy values for the different registration methods and the dependency on visual areas. Error bars denote standard deviation. (d) Reproducibility of the visual area dependency of mean entropy.

A

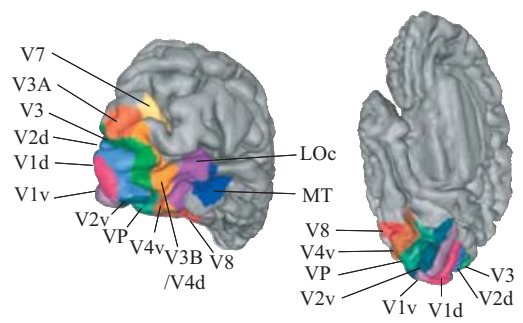

C
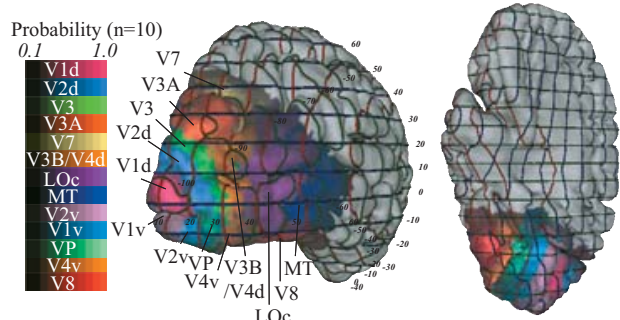

$E$

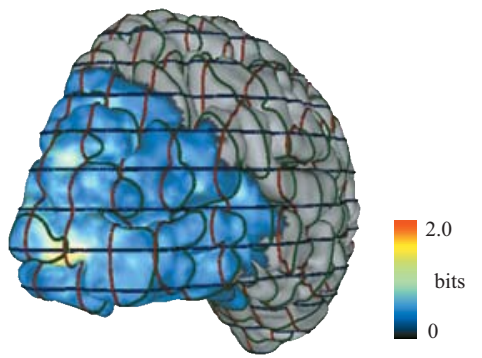

B

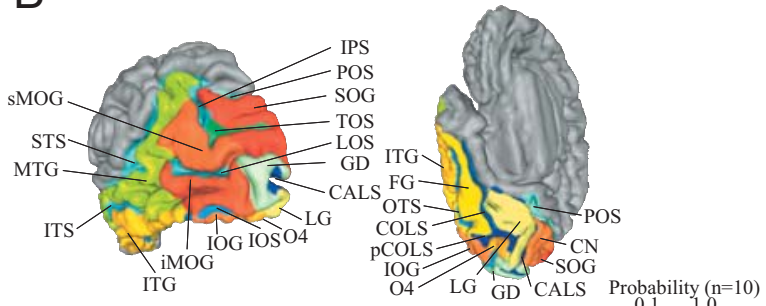

D

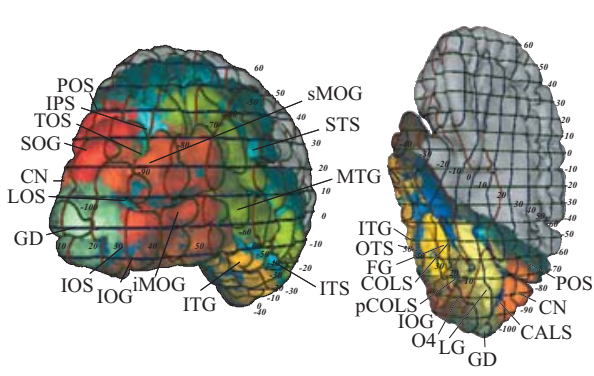

$\mathrm{F}$

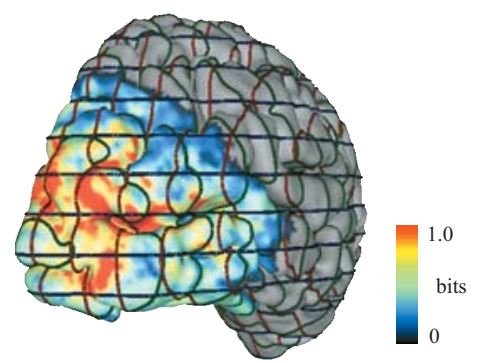

CALS 
Despite some drawbacks, the absolute entropy values obtained in this study will be useful. We showed that entropy values in the visual areas were approximately 1 bit and thus almost the same as the uncertainty between the two choices. It is therefore essential to understand the limits of intersubject brain registration for the precise interpretation of brain functional data.

\subsection{Information-theoretic Approach for Quantifying Brain Structure-function Relationships}

The concept of entropy can be applied not only to the evaluation of brain registration, but also to the analysis of brain structure-function relationships [25]. Probabilistic maps of the human brain have generally been constructed separately for brain structure and function. Integration of structural and functional probabilities is essential to explore brain structure-function relationships. To this end, information theory is useful as it allows quantification of brain structure-function relationships, taking advantage of the mutual information of a simultaneous probability map of the structural and functional brain areas. Briefly, the principle is explained as follows. Probability maps of the brain store information on structural variability $(S)$ and functional variability $(F)$ in a population. The maps quantify the variability of the location of each structural unit $\left(s_{1}, s_{2}, \ldots, s_{n}\right)$ or functional unit $\left(f_{1}, f_{2}, \ldots, f_{n}\right)$, providing a probability $p\left(s_{i}\right)$ or $p\left(f_{j}\right)$ for each point in a standardized brain space. The procedure is to measure first the amount of uncertainty involved in $S$ and $F$, respectively, using entropy and Eq.(1), and second the amount of information that one reveals about the other. The amount of information contained in $S$ about $F$ can be estimated by the decrease in uncertainty that the observer has about $F$ after knowing $S$. Based on this concept, mutual information $I(F, S)$ between $S$ and $F$ is defined by the expression,

$$
\begin{aligned}
& I(F, S)=H(F)-H(F / S), \\
& I(F, S)=\sum_{i, j} p\left(s_{i}, f_{j}\right) \cdot \log _{2} \frac{p\left(s_{i}, f_{j}\right)}{p\left(s_{i}\right) \cdot p\left(f_{j}\right)} .
\end{aligned}
$$

Mutual information $I(F, S)$ allows us to evaluate the degree of the statistical dependency between the structural and functional organization of the brain. When there is no relation between structure and function, then $I(F, S)=0$; on the other hand, when the knowledge of structural units completely determines the functional units, then $I(F, S)=H(F)$. (Fig. 6) shows a preliminary result of this approach in which the relation between 12 visual areas and 22 major sulci/gyri after the linear volume-based registration was analyzed using mutual information. Mean mutual information between the visual areas and the sulci/gyri was 0.57 bits (SD: 0.34 ), indicating the statistical dependency between them by that amount. Notably, the spatial distribution of mutual information was not homogeneous, suggesting selective associations between structures and functions. As such, the mutual information map offers quantitative and intuitive understanding of brain structure-function relationships.

\section{CONFLICT OF INTEREST}

The author declared no conflict of interest. This work was supported by Grant-in-Aid for Scientific Research
(KAKENHI), MEXT, Japan (15730335, 25135720, 2313 $5517,22530793,15 \mathrm{H} 03464$, and 15H01764).

\section{ACKNOWLEDGEMENTS}

I would like to thank M. Fukunaga, H. Mano, T. Murase, M. Umeda, H. Yamashiro, T. Yamamoto, H. Ban, and M. Umeda for their support in performing fMRI measurements and analyses.

\section{REFERENCES}

[1] Toga AW. Brain warping. San Diego: Academic Press 1999; Vol 8, pp. 385.

[2] Yamamoto H, Fukunaga M, Takahashi S, et al. Inconsistency and uncertainty of the human visual area loci following surface-based registration: Probability and entropy maps. Hum Brain Mapp 2012; 33(1): 121-29.

[3] Yamamoto H. A probabilistic atlas of human visual areas and an information-theoretical analysis of individual variability in their loci. In: Christopoulos GI, Smirnakis SM, Eds. Advanced topics in brain neuroimaging: methods and applications. Rijeka, Croatia: INTECH 2014; pp. 219-41.

[4] Mazziotta J, Toga A, Evans A, et al. A probabilistic atlas and reference system for the human brain: International consortium for brain mapping (ICBM). Philos Trans R Soc Lond B Biol Sci 2001; 356(1412): 1293-322.

[5] Mazziotta JC, Toga AW, Evans A, Fox P, Lancaster J. A probabilistic atlas of the human brain: theory and rationale for its development. The International consortium for brain mapping (ICBM). Neuroimage 1995; 2(2): 89-101.

[6] Van Essen DC, Dierker DL. Surface-based and probabilistic atlases of primate cerebral cortex. Neuron 2007; 56(2): 209-25.

[7] Shannon CE. The mathematical theory of communication, I and II. Bell Syst Tech J; 1948; 27: 379-443.

[8] Van Essen DC. Windows on the brain: the emerging role of atlases and databases in neuroscience. Curr Opin Neurobiol 2002;12(5): 574.

[9] Engel SA, Rumelhart DE, Wandell BA, et al. fMRI of human visual cortex. Nature 1994; 369(6481): 525.

[10] Sereno MI, Dale AM, Reppas JB, et al. Borders of multiple visual areas in humans revealed by functional magnetic resonance imaging. Science 1995; 268(5212): 889-93.

[11] DeYoe EA, Carman GJ, Bandettini P, et al. Mapping striate and extrastriate visual areas in human cerebral cortex. Proc Natl Acad Sci USA 1996; 93(6): 2382.

[12] Engel SA, Glover GH, Wandell BA. Retinotopic organization in human visual cortex and the spatial precision of functional MRI. Cereb Cortex 1997; 7(2): 181-92.

[13] Ejima $\mathrm{Y}$, Takahashi $\mathrm{S}$, Yamamoto $\mathrm{H}$, et al. Interindividual and interspecies variations of the extrastriate visual cortex. Neuroreport 2003;14(12):1579-83.

[14] Yamamoto H, Ban H, Fukunaga M, Umeda M, Tanaka C, Ejima Y. Large- and small-scale functional organization of visual field representation in the human visual cortex. In: Portocello TA, Velloti RB, Eds. Visual Cortex: New Research. New York: Nova Science Publisher 2008: pp. 195-226.

[15] Talairach J, Tournoux P. Co-planar stereotaxic atlas of the human brain: 3-dimensional proportional system: an approach to medical cerebral imaging. Stuttgart; New York: Thieme ;Thieme Medical 1988; Vol.8, pp 122.

[16] Desmond JE, Lim KO. On- and offline Talairach registration for structural and functional MRI studies. Hum Brain Mapp 1997; 5(1): 58-73.

[17] Smith SM. Fast robust automated brain extraction. Hum Brain Mapp 2002;17(3):143-55.

[18] Holmes CJ, Hoge R, Collins L, Woods R, Toga AW, Evans AC. Enhancement of MR images using registration for signal averaging. J Comput Assist Tomogr 1998; 22(2): 324-33.

[19] Woods RP, Grafton ST, Watson JD, Sicotte NL, Mazziotta JC. Automated image registration: II. Intersubject validation of linear and nonlinear models. J Comput Assist Tomogr 1998; 22(1): 153.

[20] Fischl B, Sereno MI, Tootell RB, Dale AM. High-resolution intersubject averaging and a coordinate system for the cortical 
surface. Hum Brain Mapp 1999; 8(4): 272-84.

[21] Dale AM, Fischl B, Sereno MI. Cortical surface-based analysis. I. Segmentation and surface reconstruction. Neuroimage 1999; 9(2): 179.

[22] Yamamoto H, Ed. BAVIEW: software for visualization and analysis of probabilistic atlases of human visual areas. ESMRMB 2011; Leipzig/DE

[23] Henriksson L, Karvonen J, Salminen-Vaparanta N, Railo H, Vanni S. Retinotopic maps, spatial tuning, and locations of human visual areas in surface coordinates characterized with multifocal and blocked FMRI designs. PloS one 2012;7(5): e36859.

[24] Hinds OP, Rajendran N, Polimeni JR, et al. Accurate prediction of

V1 location from cortical folds in a surface coordinate system. Neuroimage. 2008; 39(4): 1585-99.

[25] Yamamoto H, Fukunaga M, Tanaka C, Ebisu T, Umeda M, Ejima Y, Eds. A new method for quantifying brain structure-function relationships based on simultaneous probability map and information theory. Society for Neuroscience 33rd Annual Meeting; 2003; New Orleans. 